\title{
Fast-track pathways for drug approvals: the Australian experience so far
}

\begin{abstract}
Adrian Bootes
Assistant secretary, Head'

Josephine Maundu

Policy officer'

Sarah Golding

Medical officer ${ }^{1}$

\section{Mark McDonald}

Senior principal research scientist ${ }^{1}$

\section{John Lombard \\ Scientific writer ${ }^{2}$}

'Prescription Medicines Authorisation Branch

${ }^{2}$ Regulatory Education and Compliance Branch

Health Products Regulation Group, Australian Government Department of Health, Canberra
\end{abstract}

\section{Keywords}

drug regulation,

Therapeutic Goods

Administration

Aust Prescr 2019;42:118-9 https://doi.org/10.18773/ austprescr.2019.044
In 2016, the Australian Government announced that the Therapeutic Goods Administration (TGA) would establish two new pathways for the rapid approval of therapeutic advances and life-saving drugs.' These are the priority review and provisional approval pathways. The aim is to make the medicines available to the people who need them sooner than the usual regulatory process.

A drug is only eligible for a fast-track pathway if its proposed primary indication is for the treatment, prevention or diagnosis of a life-threatening or seriously debilitating condition. The drug must also represent a major therapeutic advance in safety or efficacy relative to already approved treatments. In addition to new drugs, new indications are eligible for the fast-track pathways.

The priority review pathway aims to complete the evaluation of a full set of data in 150 working days rather than the 255 legislated working days for a standard approval. A priority review requires a complete dossier based on clinical trial data, just like the standard approval process. For this reason, if the priority review is successful, the drug receives full registration on the Australian Register of Therapeutic Goods.

From January 2018 to July 2019, the TGA approved applications for 64 new chemical entities and 98 extensions of indications. Up to July 2019, 15 applications had been approved by priority review, including four new chemical entities and 12 extensions of indications. Twelve of these approvals were for drugs used in cancers where existing therapies have very limited efficacy. The time taken for priority reviews ranged from 80 to 147 working days with an average of 113 working days.

Before a drug can be evaluated under priority review, its sponsor, usually a pharmaceutical company, must apply for a determination that the drug is eligible for this pathway. An example of how the TGA makes a determination is alectinib for lung cancer.

Alectinib was already registered on the Australian Register of Therapeutic Goods for the treatment of patients with anaplastic lymphoma kinase (ALK)positive, locally advanced or metastatic non-small cell lung cancer. However, it was only approved for patients who had progressed on or were intolerant of crizotinib. The sponsor sought a new primary indication, that would extend the use of alectinib to first-line treatment, and for this to be considered for priority review.

The sponsor provided a clinical rationale for approving the new indication based on a phase III randomised trial. This compared alectinib to crizotinib in patients with untreated ALK-positive non-small cell lung cancer. The trial found that alectinib had a significant benefit on the primary efficacy outcome (progression-free survival), particularly in the central nervous system. Alectinib also had a better overall safety profile than crizotinib. The findings of the trial constituted substantial evidence that alectinib would be a major therapeutic advance compared to crizotinib.

The TGA determined that the submitted clinical data made the new indication eligible for priority review. After evaluation of the complete dossier, this indication for alectinib was approved for full registration on the Australian Register of Therapeutic Goods. ${ }^{2}$

The provisional approval pathway, in contrast to the priority review pathway, enables a time-limited registration of a promising drug, based on preliminary (usually phase II) clinical data. ${ }^{3}$ If approved, the drug will be available for two years and the drug's sponsor can apply for extensions up to a total of six years. Typically, the final stages of clinical trials (phase III) that address the safety, quality and efficacy of a medicine can take several years. By accepting applications for assessment before these trials are completed, a medicine could be brought to market potentially years sooner than under previous processes.

Up to July 2019, 13 applications had been determined as eligible for the provisional approval pathway. The first approval using this pathway was for an extension of indications for pembrolizumab.

The TGA only grants provisional approval if the potential benefit of early availability outweighs the risks of incomplete data about the drug. For example, a drug may be potentially life-saving, but if trial data on morbidity or mortality are not available, or the results are based on surrogate end points that have not been shown to reliably predict clinical benefit, it may not be eligible for this pathway. 
As a condition of provisional approval, the sponsor must provide a Risk Management Plan (required for all new chemical entities and major extensions of indication) and submit comprehensive clinical safety and efficacy data within the provisional time period and in accordance with this plan. If the sponsor does not follow this plan, they will not meet their conditions of registration and the TGA may revoke or not extend the drug's provisional registration. Successful evaluation of these confirmatory data will result in a transition to full registration on the Australian Register of Therapeutic Goods. The initial provisional approval and continuing registration is only permitted if the TGA is satisfied that confirmatory data will be submitted within six years.

Postmarketing surveillance of all prescription drugs is ongoing and there have been recent updates to enhance our postmarket monitoring and compliance framework. As part of these changes, provisionally registered medicines will be given high priority for postmarketing surveillance activities. As of January 2018, new drugs and new indications are marked with a black triangle symbol $\boldsymbol{\nabla}$ on the Product
Information and Consumer Medicines Information. ${ }^{4}$ This is a reminder to report adverse events that may be associated with the drug as this reporting continues to be a valuable supplement to information from sponsors and other international regulators. It does not mean that there are known safety problems, only that the TGA encourages prescribers, sponsors, pharmacists and patients to report adverse events as the medicine is new, so it can build a full picture of the drug's safety profile. This is particularly the case for provisionally registered drugs.

The eligibility criteria for both of these fast-track pathways ensure that they are restricted to those drugs that patients need most urgently. Adverse event reports help the TGA to monitor the safety of these medicines, particularly when they are new to market. The pathways increase the options for patients with life-threatening or debilitating conditions, while maintaining Australia's strong national standards for safety, quality and efficacy. $<$

The authors are either current or former employees of the Therapeutic Goods Administration.

\section{REFERENCES}

1. Therapeutic Goods Administration. MMDR: Prescription medicines regulatory reforms. Canberra: Department of Health; 2018. https://www.tga.gov.au/hubs/mmdr/ mmdr-prescription-medicines-regulatory-reforms [cited 2019 Jul 1]

2. Therapeutic Goods Administration. AusPAR: Alectinib. Canberra: Department of Health; 2018. https://www.tga.gov.au/ auspar/auspar-alectinib [cited 2019 Jul 1]
3. Therapeutic Goods Administration. Provisional determination eligibility criteria. Supporting documentation requirements. Canberra: Department of Health; 2018. https://www.tga.gov.au/publication/provisional-determinationeligibility-criteria\#supporting [cited 2019 Jul 1]

4. Therapeutic Goods Administration. Black Triangle Scheme. Canberra: Department of Health; 2018. www.tga.gov.au/ black-triangle-scheme [cited 2019 Jul 1]

\section{FURTHER READING}

Therapeutic Goods Administration. Pharmacovigilance Inspection Program. Canberra: Department of Health; 2019. www.tga.gov.au/ pharmacovigilance-inspection-program. [cited 2019 Jul 1]

Therapeutic Goods Administration. TGA online: Adverse event reporting [online form]. https://aems.tga.gov.au [cited 2019 Jul 1]

Therapeutic Goods Administration. Reporting adverse events.

Canberra: Department of Health; 2018. www.tga.gov.au/

reporting-adverse-events [cited $2019 \mathrm{Jul} 1]$
Martin J, Shenfield G. The hazards of rapid approval of new drugs. Aust Prescr 2016;39:2-3. https://doi.org/10.18773/ austprescr.2016.005

Kubler P. Fast-tracking of new drugs: getting the balance right. Aust Prescr 2018;41:98-9. https://doi.org/10.18773/ austprescr.2018.032 